\title{
The Influence Factors of Medical Disputes in Shanghai and Implications - From the Perspective of Doctor, Patient and Disease (Observational Study)
}

Yu Liu

Shanghai Normal University

Fei Yu

Shanghai Tenth People's Hospital

Feng Zhao

Shnaghai Changhai Hongkou Hospital

Xingchen Yang

Shanghai Changzheng Hospital

Guangyao Ji

Shanghai Tenth Hospital

Xiao Pan

Shanghai Changzheng Hospital

Feng Leng

Shanghai Changzheng Hospital

Dongdong Dan

Shanghai Changzheng Hospital

Pei Wang

East China Normal University

Yonghai Bai (D Baiyonghai179@126.com )

Shanghai Changzheng Hospital https://orcid.org/0000-0001-5818-7216

\section{Research}

Keywords: Medical Dispute, Dispute Level, Factor of Doctor, Factor of Patient, Factor of Disease

Posted Date: January 18th, 2021

DOl: https://doi.org/10.21203/rs.3.rs-147013/v1

License: (c) (i) This work is licensed under a Creative Commons Attribution 4.0 International License.

Read Full License 


\section{Abstract}

Objective Based on the cases collected in eight hospitals in Shanghai in recent three years, causes of medical disputes in Shanghai and influence factors of medical dispute levels were discussed, and targeted suggestions were put forward.

Methods Multistage sampling were used to collect 561 cases of medical disputes occurred in two Class A Tertiary hospitals, two Class A Secondary hospitals and four community hospitals in Shanghai in recent three years. On the basis of questionnaire, the causes of medical disputes were analyzed by descriptive statistics and the factors affecting the level of medical dispute were analyzed by means of one-way ANOVA and Logistic regression analysis.

Results Factors of doctor and patient are involved in the causes of medical disputes, with $87.1 \%$ disputes related to doctors and $13.9 \%$ related to patients. The doctor's factors include lack of communication (28.82\%), low technical level (16.91\%), lack of sense of responsibility (8.86\%), defective case records (6.92\%), imperfect operation (6.44\%), inadequate experience (6.44\%), inadequate condition evaluation (5.8\%), irregular management process $(4.03 \%)$, violation of diagnosis and treatment regulation $(4.03 \%)$, misdiagnosis and mistreatment $(3.54 \%)$, belated diagnosis and treatment $(2.58 \%)$, postoperative complications $(2.42 \%)$, equipment problems $(1.13 \%)$, missed diagnosis $(0.81 \%)$, poor condition monitoring $(0.48 \%)$, unreasonable charge $(0.48 \%)$ and poor service attitude $(0.32 \%)$. The patient's factors include misunderstanding of medical behavior (43.48\%), high expectation of prognosis (25\%), bad attitude (13.04\%), inadequate medical knowledge (7.61\%), disturbance $(6.52 \%)$, poor compliance $(3.62 \%)$ and mistrust (1.09\%). Among all medical disputes, there are 406 cases of level-4 medical disputes (78\%), 95 cases of level-3 medical disputes (18\%), 19 cases of level-2 medical disputes (4\%), and no level-1 medical dispute. Meanwhile, the classification of diseases, treatment effect, doctors' violation of diagnosis and treatment regulation, and low technical level are the reasons for the level differences in medical disputes.

Conclusion Factors of doctor in medical disputes in Shanghai mainly include inadequate communication and low technical level while the patient's factors mainly contain misunderstanding of medical behavior and high expectation of the prognosis. Level-3 and 4 medical disputes take up the major part in all medical disputes. The classification of diseases, treatment effect, doctors' violation of diagnosis and treatment regulation, and doctor's deficient technical level are high-risk factors requiring critical attention in medical disputes.

\section{Introduction}

Doctor-patient relationship is the core of medical interpersonal relationship and crucial to health-care delivery. It is regarded as the interaction between a care provider and a care user. ${ }^{[1]}$ Medical disputes generally refer to dissent between patients' families and medical care providers on medical results and reasons causing such results ${ }^{[2]}$. The doctor-patient relationship is generally harmonious in developed 
countries such as Britain and the United States as these countries own relatively comprehensive legal system and medical insurance system. However, there are still a high proportion of conflicts in medical institutions. Health professionals in the UK, especially general practitioners (GPs), are consistently at risk of work place violence, with around $10-11 \%$ of GPs reportedly experiencing assault, $5 \%$ threats with a weapon, and $25-59 \%$ verbal abuse. In addition, $63.7 \%$ of sampled Australian GPs had experienced some violence at work ${ }^{[3]}$. In 2005, a survey by Michigan Medicine on workplace violence targeted on emergency physicians revealed that $74.9 \%$ of physicians suffered verbal threats at least once a year, and $28.1 \%$ of physicians suffered physical violence within a year ${ }^{[4]}$; In developing countries and countries undergoing social transformation, survey on health department staff showed that more than half of the medical staff had experienced physical or psychological violence for at least one time ${ }^{[5]}$. Such facts indicate that conflict between doctor and patient has become a global issue ${ }^{[6]}$.

In China, the number of medical disputes surges since the beginning of the 21 st century due to the mismatch of supply and demand in health services and patients' rising awareness of rights as well as imperfect legal system. ${ }^{[7]}$ Since the implementation of Medical Malpractice Management Regulation in September 2002, medical disputes have increased by an annual rate of $22.9 \%{ }^{[8]}$, and even as high as $40 \%$ in some regions ${ }^{[9]}$. There have been many vicious killings targeted on doctors ${ }^{[10]}$. According to data released by National Health Commission of China, the number of outpatients in medical institutions nationwide was 73 million in 2015, of which about 70,000 people ended in medical disputes. In 2017, there were 12,734 cases of medical dispute, which decreased slightly to 12,249 in 2018 and bounced back to 18,112 in $2019^{[11]}$. In recent years, medical disputes caused by medical negligence are rising year by year. More remarkably, the cause is shifting from medical negligence to non-medical negligence. It becomes more difficult to predict and handle the disputes and the amount of compensation is getting higher and higher, resulting in a general mistrust crisis between doctors and patients ${ }^{[12]}$. The disharmony between doctors and patients should not merely be a simple medical issue but a social focus that needs keen concern ${ }^{[13]}$. Medical disputes must be greatly emphasized on as they damage medical practice environment and are not conducive to protecting patients' life and health and are discordant with the construction of harmonious society. Studying the influence factors of medical disputes to prevent and control disputes will help hospitals maintain stable operation, reduce expenditure, improve work efficiency and economic benefits, and alleviate medical personnel's fear and anxiety on medical disputes to arouse their enthusiasm and creativity. Therefore, we must strengthen research on medical disputes in order to discover the potential or existing risks in medical services in time, provide basis for pre-control of medical risks, ensure sound operation of hospitals, maintain common interests between doctors and patients and improve the quality of medical services. Deepening the research on doctor-patient relationship can prevent conflicts and reduce possible negative consequences in the short term, and strengthen humanistic care and create a harmonious environment in the long term.

As an international financial center, Shanghai is one of the cities with the richest medical resources in China, with its number of medical disputes increasing at an annual rate of $11 \%{ }^{[14]}$. Domestic scholars mainly study doctor-patient relationship in four aspects, namely doctor-patient relationship research from 
the perspective of different disciplines, research on the nature of doctor-patient relationship, investigation of doctor-patient relationship, research on influence factors of doctor-patient relationship, and research on countermeasures for building harmonious doctor-patient relationship. Foreign scholars, on the other hand, concentrate on discussing the modes of doctor-patient relationship ${ }^{[15]}$. Innovation of this paper is that taking hospital management as the cut-in point and based on the perspectives of doctors, patients and diseases, 561 cases of medical disputes occurred in Shanghai in recent three years were extracted by multistage sampling. High-risk factors of disputes were analyzed and the correlation test on influence factors that would further escalate medical disputes was undertaken. It will provide a basis for hospitals in Shanghai to prevent medical disputes and lay a foundation for further establishment of early intervention indicators for medical disputes.

\section{Methods}

\subsection{Subjects}

561 medical disputes occurred in two Class A Tertiary hospitals, two Class A Secondary hospitals and four community hospitals in Shanghai in recent three years were collected via multistage sampling, among which 41 cases were removed due to imperfect information processing and 520 cases with complete information were finally included. The pass rate was $92.69 \%$.

\subsection{Research tools}

The research group had developed the Questionnaire on Medical Dispute Case Analysis ${ }^{[16]}$ in early stage, which was divided into 6 dimensions and 23 items, including demographic indicators ( 6 items), medical factors ( 4 items), patient factors ( 2 items), disease factors ( 4 items), communication factors ( 2 items) and dispute handling factors (5 items).

\subsection{Procedures}

Retrospective analysis was performed on cases respectively and documents were processed. Before the investigation, hospital president in charge of medical disputes were requested to coordinate and the questionnaires were filled in by trained investigators uniformly. During the process, questions were answered in time to guarantee the authenticity and effectiveness of the investigation. Questionnaires were collected and checked upon interviewees finishing the survey. Interviewees were asked to complete the questionnaire if there was any omission. Data collected in the questionnaire was recorded into Excel and processed by SPSS then.

\section{Results And Analysis}

\subsection{Analysis on Influence Factors of Medical Disputes}

\subsubsection{Factors of doctor}


The doctor's factors mainly included insufficient communication, low technical level, lack of sense of responsibility, defective case records, imperfect operation, inadequate experience, inadequate condition evaluation, irregular management process, violation of diagnosis and treatment regulation, misdiagnosis and mistreatment, belated diagnosis and treatment, and postoperative complications (see Table 1).

Table 1

Doctor's factors in medical disputes

\begin{tabular}{|lll|}
\hline Reason & Number of conflict (case) & Proportion (\%) \\
\hline insufficient communication & 179 & 28.82 \\
\hline low technical level & 105 & 16.91 \\
\hline lack of sense of responsibility & 55 & 8.86 \\
\hline defective case records & 43 & 6.92 \\
\hline imperfect operation & 40 & 6.44 \\
\hline inadequate experience & 40 & 6.44 \\
\hline Inadequate condition evaluation & 36 & 5.80 \\
\hline irregular management process & 25 & 4.03 \\
\hline violation of diagnosis and treatment regulation & 25 & 4.03 \\
\hline misdiagnosis and mistreatment & 22 & 3.54 \\
\hline belated diagnosis and treatment & 16 & 2.58 \\
\hline postoperative complications & 15 & 2.42 \\
\hline equipment problems & 7 & 1.13 \\
\hline missed diagnosis & 5 & 0.81 \\
\hline poor condition monitoring & 3 & 0.48 \\
\hline unreasonable charge & 3 & 0.48 \\
\hline poor service attitude & 2 & 0.32 \\
\hline
\end{tabular}

As is shown in Table 1, the main reasons for hospitals in medical disputes contained insufficient communication, low technical level, lack of sense of responsibility, defective case records, imperfect operation, insufficient experience, and inadequate condition evaluation. Lack of communication and low technical level summed up $45.73 \%$ of the influence factors in medical disputes, which should be the priority to be handled to alleviate contradiction between doctors and patients.

\subsubsection{Factors of patient}


The patient's factors mainly included misunderstanding of medical behavior, high expectation for prognosis, mistrust, and patient's speech threatening doctor. There were 40 cases where patients misunderstood medical behavior, accounting for $24.7 \%$; 23 cases with high expectation of disease prognosis, accounting for $13.3 \%$; 1 case of mistrust in doctors, accounting for $0.6 \%$; 6 cases of disturbance, accounting for $3.8 \%$; and 5 cases of patient's speech threatening doctor, accounting for $3.16 \%$ (see Table 2).

Table 2

Patient's factors in medical disputes

\begin{tabular}{|lll|}
\hline Reason & Number of conflict (case) & Proportion (\%) \\
\hline misunderstanding of medical behavior & 40 & $43.48 \%$ \\
\hline high expectation of prognosis & 23 & $25.00 \%$ \\
\hline bad attitude & 12 & $13.04 \%$ \\
\hline inadequate medical knowledge & 7 & $7.61 \%$ \\
\hline disturbance & 6 & $6.52 \%$ \\
\hline poor compliance & 3 & $3.26 \%$ \\
\hline mistrust & 1 & $1.09 \%$ \\
\hline
\end{tabular}

From Table 2, it can be seen that misunderstanding of medical behavior and high expectation for prognosis took up $68.48 \%$ and were the main reasons for medical disputes.

\subsubsection{Factors of disease}

Factors of disease mainly included disease classification and the treatment effect. Diseases were divided into four categories in line with the principle of case classification ${ }^{[17]}$, namely, simple general cases, simple emergent cases, complex intractable cases and complex critical cases.

From the perspective of disease classification, 300 cases were simple general cases, which occupied $57.69 \%$; 47 were simple emergent cases, which occupied 9.04\%; 115 were complex intractable cases, which occupied $22.12 \%$; and 58 were complex critical cases, which occupied $11.15 \%$ (see Table 3 ). Simple general cases took up $57.69 \%$ and possessed highest proportion in medical disputes. 
Table 3

Distribution of disease factors in medical disputes

\begin{tabular}{|lll|}
\hline Disease classification & Number of case & Proportion (\%) \\
\hline Simple general & 300 & 57.69 \\
\hline Simple emergent & 47 & 9.04 \\
\hline Complex intractable & 115 & 22.12 \\
\hline Complex critical & 58 & 11.15 \\
\hline Treatment effect & & \\
\hline Clinically cured & 69 & 20.25 \\
\hline Remission & 148 & 21.52 \\
\hline Aggravation & 152 & 24.68 \\
\hline Death & 151 & 33.54 \\
\hline
\end{tabular}

From the perspective of treatment effect, 69 cases causing medical disputes were clinically cured cases, which accounted for 20.25\%; 148 were remission cases, which accounted for $21.52 \%$; 152 were aggravation cases, which accounted for $24.68 \%$; 151 were death cases and accounted for $33.54 \%$ (see Table 3). The proportion of medical disputes caused by patient's death was the highest, owning $33.54 \%$.

\subsection{Analysis on Influence Factors of Medical Dispute Level 3.2.1 Medical dispute level}

Medical disputes are classified into four levels: level-4 medical disputes with compensation below 100,000 RMB, level-3 medical disputes with compensation between 100,000 and 500,000 RMB, level-2 medical disputes with compensation between 500,000 and one million RMB, and level-1 medical disputes with compensation above one million RMB ${ }^{[18]}$. Among the 520 cases of medical disputes sampled by multistage sampling, 406 cases belonged to level- 4 medical disputes, which were $78 \%$; 95 cases were level-3 medical disputes and took up 18\%; 19 cases were level-2 medical disputes and accounted for $4 \%$. And there were no level-1 medical dispute. Most of the medical disputes happened in Shanghai were level-3 and level-4 medical disputes and were controllable.

\subsubsection{One-way ANOVA on influence factors of medical disputes}

Taking disease factors, factors of doctor and factors of patient as independent variables and dispute levels as dependent variables, non-parametric one-way ANOVA in SPASS was performed in analyzing 
levels of medical dispute. Results were then substituted into multiple Logistic regression model to get the indicators of high-risk factors of medical disputes.

Disease factors included disease classification and treatment effect. Factors of doctor included the doctor's professional title, working years, qualifications, violation of diagnosis and treatment regulation, misdiagnosis and mistreatment, belated diagnosis and treatment, imperfect operation, insufficient condition evaluation, low technical level, inadequate experience, defective case records, etc. Factors of patient included the patient's gender, age, native place, misunderstanding of medical behavior, high expectation of prognosis, bad attitude, inadequate medical knowledge, poor compliance, mistrust and disturbance. The patient's native place $\left(X^{2}=12.60, P=0.002\right)$, disease classification $\left(X^{2}=55.861, P=\right.$ $0.000)$, treatment effect $\left(X^{2}=80.744, P=0.000\right)$, doctor's professional title $\left(X^{2}=9.061, P=0.011\right)$, violation of diagnosis and treatment regulation $\left(X^{2}=66.956, P=0.000\right)$, belated diagnosis and treatment $\left(X^{2}=\right.$ $16.123, P=0.000)$, insufficient condition evaluation $\left(X^{2}=19.101, P=0.000\right)$, inadequate experience $\left(X^{2}=\right.$ 50.838, $P=0.000)$, low technical level $\left(X^{2}=14.916, P=0.001\right)$ and defective case records $\left(X^{2}=12.950, P=\right.$ 0.002 ) were verified by Kruskal-Wallis $\mathrm{H}$ test and the difference was statistically significant.

\subsubsection{Logistic Regression Analysis of Medical Dispute Level}

The above one-way ANOVA results indicated that the patient's native place, disease classification, treatment effect, doctor's professional title, violation of diagnosis and treatment regulation, belated diagnosis and treatment, insufficient condition evaluation, inadequate experience, low technical level and defective case record were related to the level of medical dispute. The above results of non-parametric one-way ANOVA were substituted into multiple Logistic regression equation, and the significance level was 0.05 . The following results are obtained (see Table 4). 
Table 4

Multiple Logistic regression analysis of factors influencing medical dispute level

\begin{tabular}{|llllll|}
\hline & B & SE & Wald & df & Sig \\
\hline Level-2 medical dispute & -.831 & .944 & .776 & 1 & .378 \\
\hline Level-3 medical dispute & 1.595 & .946 & 2.840 & 1 & .092 \\
\hline Disease classification (simple general) & .915 & .374 & 6.000 & 1 & .014 \\
\hline Disease classification (simple emergent) & .342 & .500 & .468 & 1 & .494 \\
\hline Disease classification(complex intractable) & -.155 & .365 & .182 & 1 & .670 \\
\hline Disease classification (complex critical) & $0^{\mathrm{a}}$ &. &. & 0 &. \\
\hline Treatment effect (cured) & 2.252 & .645 & 12.178 & 1 & .000 \\
\hline Treatment effect (remission) & 1.804 & .391 & 21.268 & 1 & .000 \\
\hline Treatment effect (aggravation) & .805 & .299 & 7.227 & 1 & .007 \\
\hline Treatment effect (death) & $0^{\mathrm{a}}$ &. &. & 0 &. \\
\hline Violation of diagnosis and treatment regulation & 1.174 & .452 & 6.752 & 1 & .009 \\
\hline Not violating diagnosis and treatment regulation & $0^{\mathrm{a}}$ &. &. & 0 &. \\
\hline Low technical level & .674 & .279 & 5.861 & 1 & .015 \\
\hline High technical level & $0^{\mathrm{a}}$ &. &. & 0 &. \\
\hline Link function: Logit. & & & & & \\
\hline a. Parameter is set as zero as it is redundant. & & & & & \\
\hline
\end{tabular}

We can see from Table 4 that the classification of diseases, the treatment effect, doctor's violation of diagnosis and treatment regulation, and low technical level were the reasons for level differences in medical disputes.

\section{Discussion}

\subsection{Influence of doctor and implications}

\subsubsection{Enhance professional training and improve clinical skills}


The medical disputes caused by doctors' deficient clinical practice (such as misdiagnosis and mistreatment, belated diagnosis and treatment, imperfect operation, inadequate condition evaluation, low technical level and insufficient experience) accounted for $36 \%$ of the total medical disputes. This is because young doctors are lack of standardized professional training and have deficient clinical practice, which lead to the decline of service quality ${ }^{[19]}$. Therefore, it is necessary for hospital to improve regulations and rules concerning the clinical diagnosis and treatment, actively launch standardized clinical pathway, carry out professional skills training specially for young doctors, and advance clinical practice of young doctors in order to decrease medical disputes.

\subsubsection{Improve communication skills and create a harmonious environment}

Medical disputes caused by insufficient communication took up $26.25 \%$ of the total medical disputes. Good communication serves as cornerstone for medical service as effective communication can improve health outcomes, patients' satisfaction, patients' understanding of treatment and compliance. Cultivation of effective communication skills is an important part to be a good doctor ${ }^{[20]}$. Communication is a technical problem requiring different skills at different stages of diagnosis and treatment. Hence medical service providers should strive to master good communication skills and hospital administrators should emphasize the importance of communication skills and formulate various methods to propel skills learning. They should ensure appropriate and effective training opportunities are offered for the staff to develop and improve their communication skills in order to promote sound interaction between doctors and patients. To bridge the gap between doctor and patient due to the professionalism of medicine, it is crucial to strengthen continuous education and training for medical personnel and improve their communication skills ${ }^{[21]}$.

\subsubsection{Establish quality control mechanism for case record and improve record writing}

The medical disputes caused by the quality of case records occupied $6.3 \%$ of the total medical disputes. Quality control on case record is an important feedforward control measure in medical quality management. Hospital administrative departments should formulate strict case record quality control system, implement three-level quality monitoring of case records, reinforce supervision and management of the medical treatment coversheet, enforce terminal case record review, conduct random checks on case records from departments prone to medical disputes from time to time, and enhance young doctors' attention to case record writing so as to elevate medical service quality, improve medical record writing and reduce the occurrence of medical disputes due to case record writing [22].

\subsection{Influence of patient and implications}

Medical disputes caused by misunderstanding of medical behavior and high expectation of prognosis accounted for $68.48 \%$ of the patient's factors. Trust is regarded as the cornerstone of doctor-patient relationship in the world of medicine and a thermometer to measure the harmony between doctor and 
patient. Patients who have confidence in doctors generally have healthier behaviors, fewer symptoms, higher similar quality and more satisfactory treatment ${ }^{[23]}$. Doctor-patient trust is a type of social trust formed during medical activities. The imperfect market-oriented reform of medical system is the institutional root for the mistrust crisis, which aggravates the gap between doctors and patients, and makes patients who are in a relatively inferior position distrust medical personnel further. To protect their own rights and interests, patients have to question medical behavior at all stages of the medical process, thus impeding development of doctor-patient trust ${ }^{[24]}$. Meanwhile, China is at the period of social transformation with the trust model changing from the "acquaintance circle" to institution-based trust model. Imperfect medical system reform has also intensified the mistrust between doctors and patients ${ }^{[25]}$. Medicine is a highly specialized subject. There is a serious information asymmetry between doctors and patients, and patients often lack enough medical knowledge. In addition, the mistrust between doctors and patients inevitably leads to misunderstanding of reasonable medical behavior. People's health awareness is gradually rising and medical technology is developing rapidly as the society develops, therefore patients' expectations for treatment effect also get higher and higher. However, due to the limitations of medical technology, it is unavoidable that patients' expectations may not match the actual treatment effect ${ }^{[26]}$, which will very likely end up in medical disputes. Though patients prevail in this kind of game, it is a negative sum game that will ultimately harm the interests of patients.

Therefore, patients should recognize the limitations of medicine and understand that disease is the common enemy of doctors and patients. They should trust doctors fully and actively discuss treatment

decisions with doctors to jointly fight against disease ${ }^{[27]}$. Doctors should fully explain the treatment plan and communicate with patients to reduce their misunderstanding of reasonable medical behavior. Possible risks during treatment should be emphasized and patients' expectations should be controlled at a reasonable level.

\subsection{Influence of disease management and implications}

The classification of diseases and treatment effect are the reasons for the differences in medical dispute levels. Curing diseases and saving life are primary tasks of the hospital while restoring health is patients' ultimate expectation to the hospital ${ }^{[28]}$. When the disease's treatment effect is not ideal enough and the patients do not know limitations of medicine, it is very likely that patients will oppose doctor's treatment plan and lead to medical disputes, even with an escalation of conflicts sometimes. Therefore, doctors should attach great importance to the rescue quality of critically ill patients, treat patients in strict accordance with clinical pathways, improve the treatment effect of diseases, and inform prognosis of the disease in time; For some critically ill patients, the hospital should establish a rapid and standardized referral mechanism, and arrange referrals in time to protect patients' life and health.

\section{Conclusion}

The doctor's factors for medical disputes in Shanghai mainly include professional title, violation of diagnosis and treatment regulation, misdiagnosis and mistreatment, belated diagnosis and treatment, 
imperfect operation, insufficient condition evaluation, low technical level, lack of experience and defective case records, etc.; The patient's factors are misunderstanding of medical behavior and high expectation for the prognosis; Disease's factors contain disease classification and the treatment effect. Among them, the classification of diseases, treatment effect, doctors' violation of diagnosis and treatment regulation, and doctors' low technical level are the reasons for the differences in medical dispute levels and belong to the high-risk factors worthy of close attention. While strengthening training of clinical skills and communication skills, hospitals should also establish a quality control mechanism for case record and construct a rapid and standardized hospital referral mechanism; Patients should actively cooperate with doctors in the treatment and doctors should fully explain to and communicate with patients to alleviate their misunderstanding of reasonable medical behavior and control patients' expectations within a reasonable range. Doctors should attach great importance to the rescue quality of critically ill patients, and inform patients of prognosis in time to prevent medical disputes and deterioration. As comrades in the same ally in front of disease, doctor and patient should cooperate with each other and encourage each other to jointly fight against disease ${ }^{[29]}$.

\section{Limitations}

Due to limitation of time and resources, our research is limited to Shanghai and only eight medical institutions are sampled via multistage sampling. The research results can't be extended to Shanghai and even the whole country owing to small sample size. We need to conduct a larger survey in Shanghai in order to describe the causes of medical disputes in Shanghai and high-risk factors escalating disputes more precisely. However, our research methods and findings can offer guidance for similar type of research.

\section{Declarations}

\section{Ethics approval and consent to participate区}

Our data has not collect from human subjects, so we do not have requires ethics approval. All the authors consent to participate.

\section{Consent for publication:}

Consent

\section{Availability of supporting data》}

Our data can support our results. 


\section{Competing interests:}

No

\section{Funding:}

Major Bidding Project of National Social Science Fund of China, Project No.: 17ZDA327

\section{Author Contributions:}

Conceptualization, Yonghai Bai; Data curation, Yu Liu and Xiao Pan; Formal analysis, Yu Liu; Funding acquisition, Peng Wang and Yonghai Bai; Investigation, Yu Liu, Guangyue Ji and Dongdong Dan; Methodology, Yu Liu; Project administration, Yonghai Bai; Resources, Xingchen Yang; Software, Feng Leng; Supervision, Yonghai Bai; Writing - original draft, Yu Liu; Writing - review \& editing, Yu Liu, Feng Zhao, Fei Yu and Peng Wang.

\section{Acknowledgements:}

Not applicable

\section{References}

1. Harbishettar V, Krishna KR, Srinivasa P,Gowda M. The enigma of doctor-patient relationship. Indian J Psychiatry 2019;61:S776-81.

2. Lin-Sheng Yu,Guang-Hua Ye,Yan-Yan Fan,etc.Analysis of Forensic Autopsy in 120 Cases of Medical Disputes Among Different Levels of Institutional Settings. J Forensic Sci, September 2015, Vol. 60, No. 5 doi: 10.1111/1556-4029.12769.

3. Kai Xing , Xue Zhang, Mingli Jiao and so on.Concern about Workplace Violence and Its Risk Factors in Chinese Township Hospitals:A Cross-Sectional Study. Int. J. Environ. Res. Public Health 2016, 13, 811.

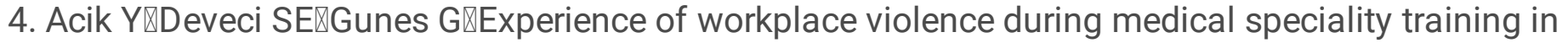
Turkey[J] $]$ Occup Med(Lond) $₫ 2008 \bowtie 58(5) \bowtie 361-366 \rrbracket$

5. Wykes T囚Publication Dissemination囚EID National Institute for Occupational Safety and Health[EB囚

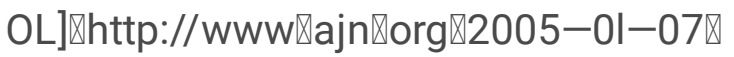

6. Morken, T.; Johansen, I.H.; Alsaker, K. Dealing with workplace violence in emergency primary health care:A focus group study. BMC Fam. Pract. 2015, 16, 51. [CrossRef] [PubMed]

7. Zeng et al. Analysis of current situation and influencing factor of medical disputes among different levels of medical institutions based on the game theory in Xiamen of China. Medicine (2018) 97:38 
8. Fei Yu ,Xiaofeng Xie,Fang Ding,Cheng Xue and Zhan Sun. Changing procedures for resolving medical disputes in China. Internal Medicine Journal 48 (2018) 1552-1553.

9. Yuan J.J., Chou X.C., Tong T.L., et al. Empirical study on methods for handling medical disputes in public hospitals [J].Journal of Shanghai Jiaotong University (Medical Science), 2016:36 (10): 15031507.

10. Yu H, Hu Z, Zhang X, Li B, Zhou S. How to overcome violence against healthcare professionals, reduce medical disputes and ensure patient safety. Pak J Med Sci 2015;31(1):4-8. doi: http://dx.doi.org/10.12669/pjms.311.6446.

11. Zhang J. Current situation and prevention measures of doctor-patient relationship in China under the new era [J].Labor Security World, 2020(12):32.

12. Wang et al. BMC Health Services Research (2020) 20:225. https://doi.org/10.1186/s12913-0205044-7区

13. Dawei Pan.Doctors Killed My Baby:Argumentative Patterns in Medical Disputes in China. HEALTH COMMUNICATION 2018, VOL.33,NO.10,1267-

1276. https://doi.org/10.1080/10410236.2017.1351271.

14. Yin L., Zeng R.H., Gao X., Gu J.D. Analysis on the status-quo and influencing factors of medical disputes in the $\square \mathrm{A}$ Hospitals--based on the perspective of doctors, patients and family members[J].Health Economics Research, 2019,36(12):67-74.

15. Yu Liu, Yonghai Bai, Pei Wang. Study of the factors causing medical disputes in a Third-Level Grade A Hospital in Shanghai[J].Int J Health Plann Manage,2018,1-10.

16. Liu Y., Bai Y.H., Xu Z.L., Wang P. Cause Analysis of Medical Disputes in a Military Hospital. Hospital Administration Journal of Chinese People's Liberation Army[J], 2018, 25(5):416-419.

17. Yu G. Design of the Case Classification System Based on XGBoost Algorithm[J]. China Digital Medicine,2018,(3): 69-71.

18. Liu Y., Bai Y.H. The influence factors of medical disputes in a tertiary hospital in Shanghai [J].Chinese Health Quality Management, 2019,26(3): 75-78.

19. Zhao S., Huang H., Wu M. Analysis of influence factors of medical disputes in a tertiary hospital from 2009 to 2014 [J]. China Medical Herald, 2016,13(8):181-184.

20. Sun et al. BMC Medical Education(2020)20:8. https://doi.org/10.1186/s12909-019-1917-z

21. Oleksii KORZH, Olga TSODIKOVA .Improving doctor-patient communication in a primary care setting. Ro J Med Pract. 2019;14(1).

22. Zhu W.J., Yu S.Y., Wang D.Z. Discussion on cultivating medical staff's doctor-patient communicative ability under the new situation [J].Soft Science of Health $₫ 2019,33(7): 68-71 \rrbracket$

23. Gu et al.The impact of contract service policy and doctor communication skills on rural patientdoctor trust relationship in the village clinics of three counties.BMC Health Services Research (2019)19:187 https://doi.org/10.1186/s12913-019-3875-x 
24. Zhang, Z.;Xiong, J.;Wu, S.The violation and repair of doctor-patient trust from the perspective of medical-Exploratory analysis based on grounded theory. System Engineering Theory and Practice,2019(5):1256-1265.

25. Li X.Q., Zhou G.P., Li Y.J., An Y.X Zhao L.B. The effective ways to implement the informed consent right in the lack of doctor-patient trust context [J]. Chinese Medical Ethics, 2019, 32(8)ष1019-1022.

26. Yan Y., Wang L.Q., Si Z.Y., Wu Y.Y., Ma D. The analysis of the causes for poor doctor-patient

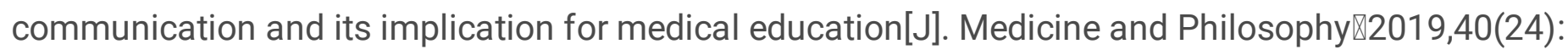
55-57.

27. Dong Wei etal.The mediating effect of trust on the relationship between doctor-patient communication and patients' risk perception during treatment.PsyCh Journal 9 (2020): 383391.DOI: 10.1002/pchj.327区

28. Yin L., Zeng R.H., Gao X., Gu J.D. Analysis on the status-quo and influencing factors of medical disputes in the $\mathbb{\triangle A}$ Hospitals--based on the perspective of doctors, patients and family members[J]. Health Economics Research, 2019,36(12):67-74.

29. Chen Z. Doctors and patients share common interests [N]. People's Daily $\llbracket 2009 \otimes 12(10) \varangle 18 \rrbracket$ 